\title{
High dietary salt intake activates inflammatory cascades via Th17 immune cells: impact on health and diseases
}

\author{
Yuvaraj Balan¹, Rajaa Muthu Packirisamy², Mohanraj P S 3
}

\begin{abstract}
${ }_{1}^{1}$ Pondicherry Institute of Medical Sciences, Kalapet, Puducherry, India

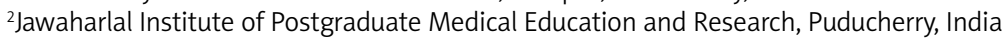
${ }^{3}$ All India Institute of Medical Sciences, Gorakhpur, India
\end{abstract}

Submitted: 6 August 2019; Accepted: 13 January 2020

Online publication: 15 June 2020

Arch Med Sci 2022; 18 (2): 459-465

DOI: https://doi.org/10.5114/aoms.2020.96344

Copyright (c) 2020 Termedia \& Banach

\section{Abstract}

The incidence of immune-mediated inflammatory diseases (IMIDs) is on the rise. A high salt content in the diet was found to play a crucial role in mediating IMIDs. It was demonstrated that increased salt concentration favors the differentiation of CD4+ cells to pathogenic Th17 cells, which predispose to several inflammatory diseases by modulating the immunological milieu. In auto-immune diseases increased salt concentration causes stable induction of Th17 cells. In cancer, increased salt concentration triggers chronic inflammation and increases vascular endothelial growth factor levels. Salt-mediated proliferation of Th17 cells has been found to reduce nitric oxide production in the endothelial cells, leading to hypertension. Increased salt concentration was found to alter the intestinal flora, which favors local inflammation. This review attempts to explain the role of high salt concentration and its molecular pathways in causing IMIDs.

Key words: cancer, inflammation, Th17 cells, immunity, sodium chloride.

\section{Introduction}

Table salt $(\mathrm{NaCl})$ is an essential component and an important ingredient in food. Salt adds taste and increases the palatability of the food. Consumption of excess amounts of salt has been implicated in various diseases including hypertension [1], heart attack [2], stroke [3], renal failure [4], and cancer [5].

The dietary salt content in South Asians is higher [6] than what is recommended by the World Health Organization (WHO), i.e. 5 g/day [7]. Although excessive use of salt has been implicated in several inflammatory diseases, the effect of a high salt diet and the molecular mechanisms in immune-mediated inflammatory diseases remain uncertain.

Immune-mediated inflammatory diseases (IMIDs) are a group of heterogeneous disorders with unknown etiology. The prevalence of IMIDs is around $5-7 \%$ and is on the rise in developing countries such as India and poses a major threat to their health care [8]. Genetic and environmental factors play a major role in the pathogenesis of IMIDs. The increase in the prevalence of IMID is attributed mainly to environmental factors such as smoking and nutritional transition, which are modifiable [9].

\author{
Corresponding author: \\ Yuvaraj Balan \\ Pondicherry Institute \\ of Medical Sciences \\ Kalathumettupathai \\ Ganapathichettikulam Village \\ No. 20 \\ Kalapet, Puducherry 605014 \\ India \\ E-mail: \\ yuvaraj613@gmail.com
}


Immune cells have a protective and preventive role against pathogens that invade the host. However, immunity is a double-edged sword; if uncontrolled it can be harmful. Kleinewietfeld et al. demonstrated that there is an increase in the differentiation of T cells to the pathogenic T cell subtype Th17 on increasing salt concentration [10]. T cells can differentiate into different lineages upon stimulation. Th1 cells produce INF- $\gamma$, which protects the host against invading intracellular pathogens. The Th2 subset produces various cytokines such as interleukin (IL)-4 and IL-5, to combat extracellular organisms [11]. In contrast, the Th17 phenotype produces inflammatory cytokines such as IL-17, tumor necrosis factor $\alpha$ (TNF- $\alpha)$, granulocyte macrophage colony-stimulating factor (GM-CSF), and enhances IL-23R expression, which are the main mediators of autoimmune diseases [12-15].

Dietary salt $(\mathrm{NaCl})$ is used abundantly in food to increase its palatability and also as a preservative in canned foods [16]. Although numerous studies have elucidated the pathogenic effect of high salt levels on various diseases such as autoimmune disorder [17-20] and hypertension, the addition of salt in these foods has become ubiquitous. In this review, insights into various possible mechanisms to show the effect of a high salt diet in mediating various diseases have been provided.

\section{High salt in the diet in auto-immune diseases}

Autoimmune diseases comprise various inflammatory diseases, such as rheumatoid arthritis (RA) and psoriasis [21]. A myriad of factors such as smoking, viral infection, stress, drugs, and pollutants have been proposed as etiological factors [22-24]. However, the primary etiologies

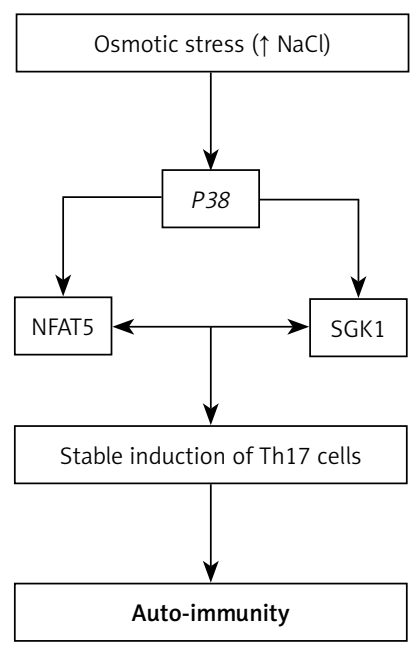

NFAT5 - nuclear factor of activated T cells 5, SGK1 - serum glucocorticoid kinase 1.

Figure 1. Role of high sodium chloride concentration in mediating autoimmune diseases of most of these autoimmune disorders are still ambiguous. The abnormal immune reaction towards the host and deposition of pathogenic Th17 cells have been demonstrated in the inflammatory sites, for instance in the skin of psoriasis patients and in multiple joints of RA patients [25-27].

The bacterial Saccharomyces cerevisiae cell wall component zymosan, fungal $\beta$-glucans, C-type lectin present in dendritic cells and pro-inflammatory cytokines such as IL-6, TNF- $\alpha$, and IL-2, were found to be the stimuli for activation of Th17 cells [28]. Interleukin 23 has been the major stimulus for sustaining and generating Th17 cells, and blocking it ameliorates the effects of Th17 cells [29].

Researchers have established an association between the increase in the incidence of autoimmune diseases and Th17 cells. However, finding the molecular switch that leads to the overproduction of Th17 cells has been a difficult endeavor. Animal studies demonstrated that mouse cells grown in high salt concentration produce a greater Th17 response than the cells grown in a normal salt concentration [30].

To explain the molecular mechanism for the above observation, the authors explored serum glucocorticoid kinase 1 (SGK1), which is known to regulate salt levels. Serum glucocorticoid kinase 1 was overexpressed in the cells grown in high salt concentration, and they confirmed the same in human cell lines. They also found a linear association between increased salt concentration and increased Th17 expression [31]

Through further investigation, Kleinewietfeld et al. elucidated that cells grown under high salt concentration express a greater amount of the $P 38$ gene [10]. The downstream target for the $P 38$ gene, the nuclear factor of activated T cells 5 (NFAT5), induces the production of IL-17 [32], which up-regulates the production of pro-inflammatory mediators [33].

All these findings suggest that a high salt diet might be the plausible environmental trigger for immune-mediated inflammatory diseases [20] (Figure 1).

\section{High salt in the diet and inflammatory bowel diseases}

Studies have shown that $17 \%$ of patients with inflammatory bowel disease also have associated immune-related diseases [34]. It is known that the lamina propria of the small intestine serves as the main residing place for immune cells [35]. The immune function of the intestine is also modified by intestinal bacteria, which was demonstrated where gut microbiome free mice showed an increased Th17 response and developed various immune-related diseases compared to normal mice [36]. Li et al. showed that the reconstitution of gut microbiome free mice with intestinal bacteria leads to suppression of the Th17 response 
and enhancement of $\mathrm{T}$ regulatory (Treg) cell function [37].

High salt consumption has a direct effect on gut microbiota and immune cells of the intestine, producing variable effects.

\section{Effect of high salt diet on gut microbiota}

A high concentration of salt in diet was found to have a direct toxic effect on gut microbiota, especially on Lactobacillus murinus. Lactobacillus murinus depleted mice were found to have an exaggerated Th17 response compared to normal mice. Oral gavage of $L$. murinus to these mice diminished the Th17 response, which confirmed that a high salt diet increases the Th17 response [37].

Recently, Maeda et al. found that intestinal Th17 cells can migrate to distant sites. For example, intestinal Th17 cells were identified in the spleen of RA patients. This confirms the role of intestinal cells in systemic immune inflammation [38].

\section{Effect of high salt diet on immune cells of the intestine}

Animal studies have shown the effect of a high salt diet on immune cells of the intestine. Mice receiving high salt tend to have exacerbated colitis and inflammatory diseases of the bowel. This was associated with an enhanced pathogenic $T$ cell response, autoantibodies, inflammatory immune mediators and a decrease in the protective immune response [39].

It has also been reported that an increase in inflammatory monocyte count and pro-inflammatory cytokines such as IL-6, IL-17, and IL-23 was seen in healthy volunteers receiving a controlled high salt diet. A significant increase in inflammatory cytokines such as TNF- $\alpha$, IL-17A, and CD4+ cells was also observed following high salt challenge [40].

To reveal the molecular mechanism behind the enhancement of the inflammatory immune response by increased salt intake, lamina propria mononuclear cells (LPMC) were grown in medium with higher salt concentration and they were found to express higher levels of pathogenic inflammatory mediators such as IL-17 and TNF- $\alpha$ along with an increase in the percentage of LPMC [41, 42].

Activated LPMC causes phosphorylation of the P38/MAPK pathway, which activates the downstream mediator NFAT5, which in turn increases the expression of pathogenic inflammatory mediators such as IL-17A and TNF- $\alpha$. High salt concentration also induces IL-23R expression, whose main function is to stabilize and increase Th17 expression [43]. All these effects lead to inflammatory disease of the bowel (Figure 2).
These results show that gut immune cells and intestinal bacteria regulate the systemic immune response. Hence it is important to note that dietary habits such as a high salt intake may be harmful and may have deleterious effects on health.

\section{High salt in the diet and hypertension}

Worldwide, increased blood pressure is one of the leading causes of mortality [44]. Hypertension leads to several life-threatening complications and has to be treated vigorously [45-47].

It has been established that salt intake is associated with hypertension, and recently it was found that immune cells grown in a high salt medium differentiate into highly pathogenic Th17 cells [48]. Interleukin 17 is the inflammatory marker produced by Th17 cells and is associated with hypertension. Interleukin 17 acts on endothelial cells and leads to eNOS Thr495 phosphorylation, which is mediated by RhoA/Rho-kinase and causes decreased NO production and diminishes the NO-mediated vasodilation [49]. All the above effects ultimately increase the systemic vascular resistance, resulting in hypertension (Figure 3).

Various studies have shown the role of IL-17 in the pathogenesis of hypertension [50]. A high salt diet also favors the polarization of CD4+ cells to Th17 cells. Recently, Kamat et al. demonstrated that mice lacking IL-17A have increased potency to excrete sodium and hence reduction in blood pressure [51]. Hence blocking the activated immune cells and inflammatory mediators produced by it can prevent hypertension. This was elucidated in an animal study where mycophenolate mofetil, a drug that is known to inhibit activa-

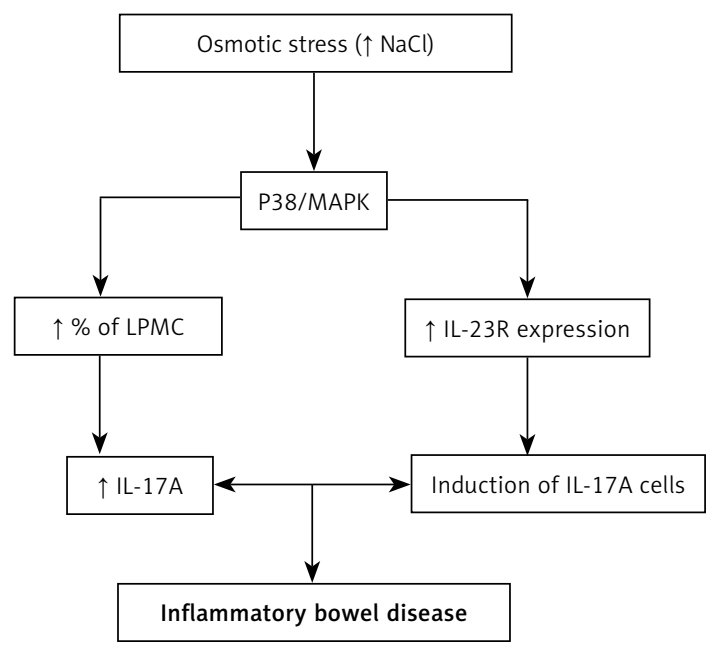

MAPK - mitogen-activated protein kinases, LPMC - lamina propria mononuclear cells.

Figure 2. Role of high sodium chloride concentration in mediating inflammatory diseases of the bowel 
tion and proliferation of immune cells, prevented the development of salt-induced hypertension in rats [52].

Machnik et al. observed that high salt-treated rats acquired a pro-inflammatory (M1) macrophage response and also exhibited water retention, leading to high blood pressure [53]. In addition to the immune-mediated mechanisms, a high salt diet was also found to modulate the renin-angiotensin-aldosterone system [54, 55] and increases sympathetic tone, thereby elevating the blood pressure [56]. Reducing salt intake in the diet could be the simplest, most cost-effective, sustainable and easiest way to prevent the development and progression of hypertension.

\section{High salt in the diet and cancer}

The effect of salt concentration on cancer development was first proposed in the 1980s, when Spars et al. initially showed that there was a high concentration of salt in human breast cancer tissue when compared to normal breast tissue [57]. Later, several studies showed a positive correlation between up-regulation of the sodium channel and tumor progression [58-60].

As discussed earlier, a high dietary salt intake causes stable induction of Th17 cells and induces inflammatory cytokines IL-1, IL-6, IL-23, TN) [61, 62]

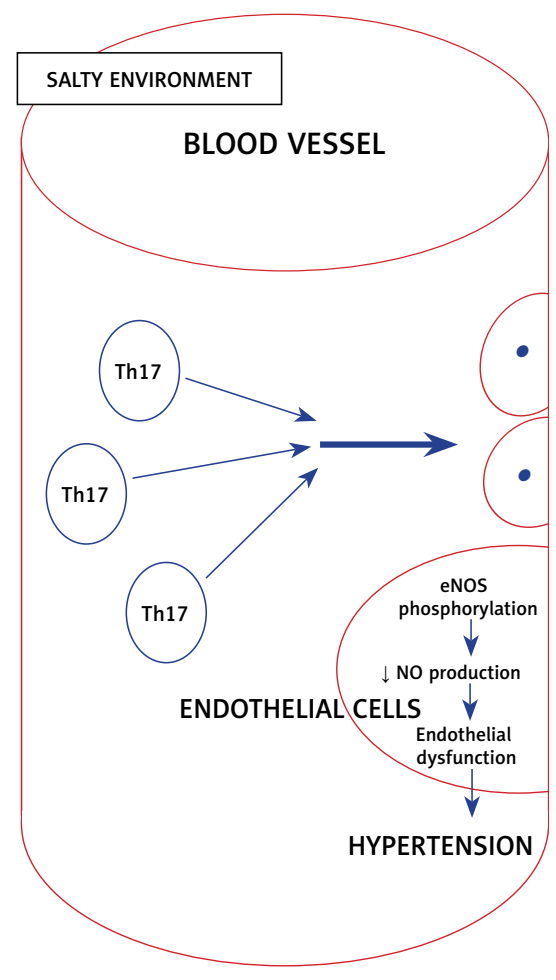

eNOS - endothelial nitric oxide synthase, $\mathrm{NO}$ - nitric oxide.

Figure 3. Role of high sodium chloride concentration in mediating hypertension and inflammatory mediators such as prostaglandins, leukotrienes, transforming growth factor (TGF), and inducible nitric oxide synthase (iNOS) $[63,64]$. Chronic induction of Th17 cells by high salt intake results in chronic inflammation [65]. Chronic inflammation exerts pleiotropic effects and is one of the important hallmark features in cancer development and progression [66].

Cells under normal environmental conditions utilize glucose in the aerobic glycolytic pathway but cells grown under a high salt environment tend to produce lactic acid, which provides a favorable environment for the cells to proliferate uncontrollably and to evade host immune defense [67]. This was confirmed by Amara et al. in a breast cancer cell line study, where the cells shifted to anaerobic metabolism under osmotic stress; this demonstrated the direct effect of high salt concentration on cancer cells [68].

Angiogenesis is one of the vital factors for the tumor to expand and survive [69]. Vascular endothelial growth factor (VEGF) is a well-recognized factor known to induce angiogenesis [70]. Recently it was demonstrated that a high salt environment induces the nuclear transcription factor NFAT5, which favors the production and expression of VEGF needed for the tumor microenvironment [71]. Apart from NFAT5, VEGF is also induced by IL-17, a hallmark proinflammatory cytokine produced by Th17 cells [72].

The immunological balance between Th17 cells and regulatory $T$ cells (Treg cells) was disturbed in the high salt environment. A high salt environment triggers an increase in the number of pathogenic Th17 cells that disturbs the immunological milieu, causing chronic inflammation. Chronic inflammation is one of the well-known predisposing factors for the development of cancer [73].

All these effects result in tumor progression (Figure 4).

The available systemic reviews [67] and prospective studies [74] indicate that there is a strong association between high salt diet and cancer risk. Hence reducing the salt content in the diet as recommended by the $\mathrm{WHO}$ [75] will reduce the disease burden in the population, and it will be a cost-effective way for disease prevention.

\section{Conclusions and future perspective}

A high salt $(\mathrm{NaCl})$ diet has various effects on the complex immune system leading to the activation of diverse inflammatory mediators which share the common inflammatory pathway. In autoimmune disorders, osmotic stress induces the activation of the stress kinase gene p38, which in turn activates several downstream mediators, notably NFAT5 and SGK1, leading to stable induction of proinflammatory Th17 cells. 


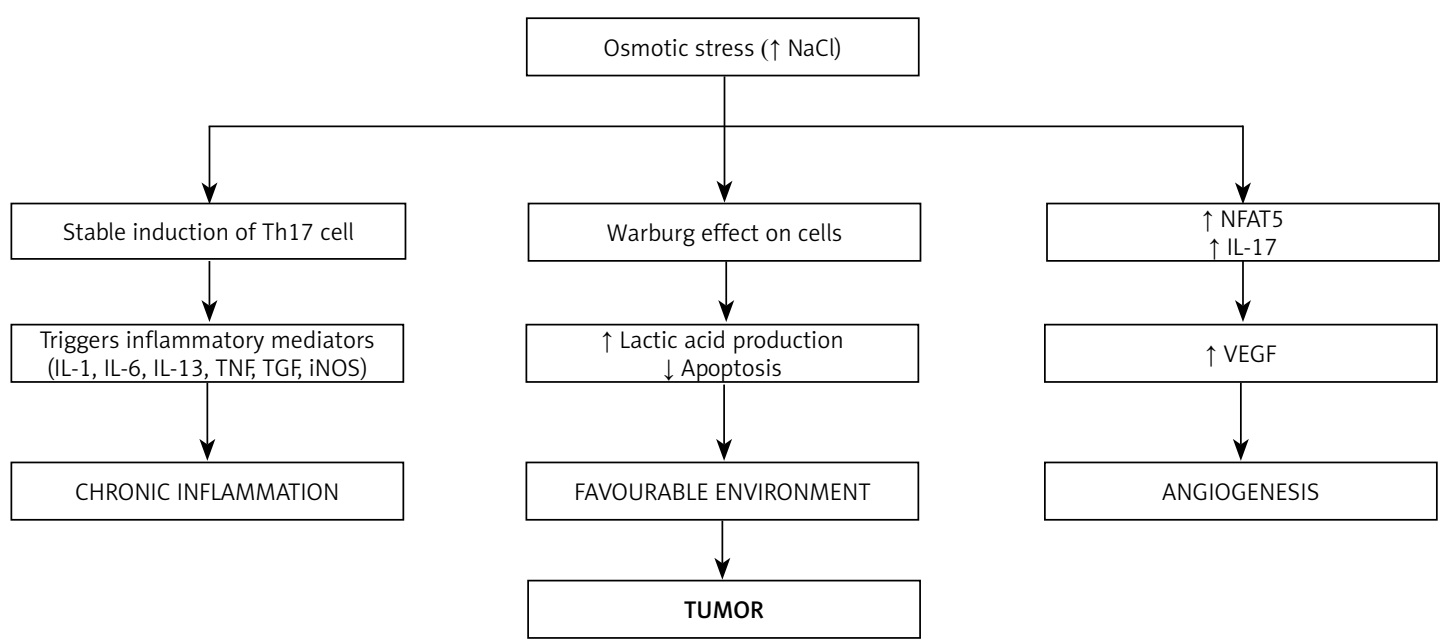

NFAT5 - nuclear factor of activated T cells 5, TNF - tumor necrosis factor, TGF - transforming growth factor, iNOS - inducible nitric oxide synthase, VEGF - vascular endothelial growth factor.

Figure 4. Role of high sodium chloride concentration in mediating cancer growth

In inflammatory bowel diseases, a high salt diet was found to affect the gut immune system as well as the microbiota. By disrupting the homeostasis in the gut, a high salt diet leads to activation of inflammatory mediators which are involved in activation and recruitment of Th17 cells.

A high salt diet affects the endothelium of blood vessels by reducing the availability of nitric oxide, mediated by IL-17, leading to the stiffening of the blood vessels. Additionally, IL-17 was found to reduce the excretion of sodium via the kidneys, resulting in its accumulation in serum. By these mechanisms, a high salt diet increases systemic vascular resistance, leading to hypertension.

A high salt diet provides a favorable environment for the tumor cells to survive and proliferate. High salt concentration in the tumor tissue favors the production of lactic acid, which protects the tumor cells from immune attack. In addition, high salt also favors angiogenesis by increasing VEGF, thereby providing nutrition to tumor cells and promoting tumor cell growth and proliferation.

Though high salt consumption has several deleterious effects on human health, lack of awareness among the public results in continued high salt usage and hence an increase in the incidence of IMIDs. Studies to explore the association between high salt usage and immune-mediated inflammatory diseases are in the initial phase or limited. By extensively studying these molecular pathways, various pharmacological drugs targeting the IMIDs can be explored. By creating awareness among the general public, the disease burden caused by excessive salt intake can be minimized.

Available evidence suggests that there is a high likelihood of a high salt diet being implicated as a risk factor for several diseases including can- cer, hypertension, and cardiovascular diseases. This article is an attempt to highlight the harmful effects of these dietary habits and would like to emphasize the importance of reducing salt content for health and disease. There is a lacuna in the literature regarding the possible mechanisms through which a high salt diet can act as a risk factor for several diseases mentioned in this article, which need further probing.

\section{Conflict of interest}

The authors declare no conflict of interest.

References

1. Dahl LK, Heine M. Effects of chronic excess salt feeding. J Exp Med 1961; 113: 1067-76.

2. Zhao $X$, Yang $X$, Zhang $X$, et al. Dietary salt intake and coronary atherosclerosis in patients with prehypertension. J Clin Hypertens 2014; 16: 575-80.

3. Appel LJ. Reducing sodium intake to prevent stroke: time for action, not hesitation. Stroke 2014; 45: 909-11.

4. Sugiura T, Takase H, Ohte N, Dohi Y. Dietary salt intake is a significant determinant of impaired kidney function in the general population. Kidney Blood Press Res 2018; 43: 1245-54.

5. Strnad M. Salt and cancer. Acta Medica Croat Cas Hravatske Akad Med Znan 2010; 64: 159-61.

6. South Asian - Action on Salt [Internet]. Available from: http://www.actiononsalt.org.uk/salthealth/ethnic/ southasian/ (Accessed: 9.05.2019).

7. Guideline: Sodium Intake for Adults and Children [Internet]. Geneva: World Health Organization; 2012. WHO Guidelines Approved by the Guidelines Review Committee. Available from: http://www.ncbi.nlm.nih.gov/ books/NBK133309/ (Accessed: 21.10.2019).

8. El-Gabalawy H, Guenther LC, Bernstein CN. Epidemiology of immune-mediated inflammatory diseases: incidence, prevalence, natural history, and comorbidities. J Rheumatol Suppl 2010; 85: 2-10. 
9. Javierre BM, Hernando $\mathrm{H}$, Ballestar E. Environmental triggers and epigenetic deregulation in autoimmune disease. Discov Med 2011; 12: 535-45.

10. Kleinewietfeld M, Manzel A, Titze J, et al. Sodium chloride drives autoimmune disease by the induction of pathogenic Th17 cells. Nature 2013; 496: 518-22.

11. Abbas AK, Murphy KM, Sher A. Functional diversity of helper T lymphocytes. Nature 1996; 383: 787-93.

12. Waite JC, Skokos D. Th17 response and inflammatory autoimmune diseases. Int J Inflamm [Internet]. 2012 Available from: https://www.ncbi.nlm.nih.gov/pmc/articles/PMC3249891/ (Accessed: 9.05.2019).

13. Robak E, Gerlicz-Kowalczuk Z, Dziankowska-Bartkowiak B, Wozniacka A, Bogaczewicz J. Serum concentrations of IL-17A, IL-17B, IL-17E and IL-17F in patients with systemic sclerosis. Arch Med Sci 2019; 15: 706-12.

14. Dańczak-Pazdrowska A, Kowalczyk M, Szramka-Pawlak B, et al. Interleukin-17A and interleukin-23 in morphea. Arch Med Sci 2012; 8: 1089-95.

15. Murdaca G, Colombo BM, Puppo F. The role of Th17 lymphocytes in the autoimmune and chronic inflammatory diseases. Intern Emerg Med 2011; 6: 487-95.

16. Intake I of M (US) C on S to RS, Henney JE, Taylor CL, Boon CS. Preservation and Physical Property Roles of Sodium in Foods [Internet]. National Academies Press (US), 2010. Available from: https://www.ncbi.nlm.nih. gov/books/NBK50952/ (Accessed: 9.05.2019).

17. Wilck N, Balogh A, Markó L, Bartolomaeus H, Müller DN. The role of sodium in modulating immune cell function. Nat Rev Nephrol 2019; 15: 546-58.

18. Haase S, Wilck N, Kleinewietfeld M, Müller DN, Linker RA. Sodium chloride triggers Th17 mediated autoimmunity. J Neuroimmunol 2019; 329: 9-13.

19. Sharif K, Amital H, Shoenfeld Y. The role of dietary sodium in autoimmune diseases: the salty truth. Autoimmun Rev 2018; 17: 1069-73.

20. Scrivo R, Perricone C, Altobelli A, et al. Dietary habits bursting into the complex pathogenesis of autoimmune diseases: the emerging role of salt from experimental and clinical studies. Nutrients 2019; 11: 1013.

21. Administrator. Autoimmune Diseases [Internet]. Australasian Society of Clinical Immunology and Allergy (ASCIA). Available from: https://www.allergy.org.au/patients/autoimmunity/autoimmune-diseases (Accessed: 9.05.2019).

22. Sharif K, Watad A, Bragazzi NL, Lichtbroun M, Amital H, Shoenfeld Y. Physical activity and autoimmune diseases: get moving and manage the disease. Autoimmun Rev 2018; 17: 53-72.

23. Perricone $C$, Versini $M$, Ben-Ami D, et al. Smoke and autoimmunity: the fire behind the disease. Autoimmun Rev 2016; 15: 354-74.

24. Sharif K, Watad A, Bragazzi NL, Adawi M, Amital $H$, Shoenfeld Y. Coffee and autoimmunity: more than a mere hot beverage! Autoimmun Rev 2017; 16: 712-21.

25. Tesmer LA, Lundy SK, Sarkar S, Fox DA. Th17 cells in human disease. Immunol Rev 2008; 223: 87-113.

26. Krueger JG, Fretzin S, Suárez-Fariñas M, et al. IL-17A is essential for cell activation and inflammatory gene circuits in psoriasis. J Allergy Clin Immunol 2012; 130: 145-54.e9.

27. Kotake S, Yago T, Kobashigawa T, Nanke Y. The plasticity of Th17 cells in the pathogenesis of rheumatoid arthritis. J Clin Med [Internet]. 2017; 6. Available from: https:// www.ncbi.nlm.nih.gov/pmc/articles/PMC5532575/ (Accessed: 9.05.2019).

28. Magee CN, Boenisch O, Najafian N. The role of co-stimulatory molecules in directing the functional differentia- tion of allo-reactive T helper cells. Am J Transplant 2012; 12: 2588-600.

29. Yu RY, Gallagher G. A naturally occurring, soluble antagonist of human IL-23 inhibits the development and in vitro function of human Th17 cells. J Immunol 2010; 185: 7302-8.

30. Dar HY, Singh A, Shukla P, et al. High dietary salt intake correlates with modulated Th17-Treg cell balance resulting in enhanced bone loss and impaired bone-microarchitecture in male mice. Sci Rep [Internet]. 2018; 8. Available from: https://www.ncbi.nlm.nih.gov/pmc/ articles/PMC5802842/ (Accessed: 9.05.2019).

31. Kleinewietfeld $M$, Hafler DA. The plasticity of human Treg and Th17 cells and its role in autoimmunity. Semin Immunol 2013; 25: 305-12

32. Lee JH, Kim M, Im YS, Choi W, Byeon SH, Lee HK. NFAT5 induction and its role in hyperosmolar stressed human limbal epithelial cells. Invest Ophthalmol Vis Sci 2008; 49: 1827-35.

33. Kuwabara T, Ishikawa F, Kondo M, Kakiuchi T. The role of IL-17 and related cytokines in inflammatory autoimmune diseases. Mediators Inflamm 2017; 2017: 3908061.

34. Weng X, Liu L, Barcellos LF, Allison JE, Herrinton LJ. Clustering of inflammatory bowel disease with immune mediated diseases among members of a northern california-managed care organization. Am J Gastroenterol 2007; 102: 1429-35.

35. McDermott AJ, Huffnagle GB. The microbiome and regulation of mucosal immunity. Immunology 2014; 142: 24-31.

36. Wilck N, Matus MG, Kearney SM, et al. Salt-responsive gut commensal modulates $\mathrm{TH} 17$ axis and disease. $\mathrm{Na}$ ture 2017; 551: 585-9.

37. Li J, Sun F, Guo Y, Fan H. High-salt diet gets involved in gastrointestinal diseases through the reshaping of gastroenterological milieu. Digestion 2019; 99: 267-74.

38. Maeda Y, Takeda K. Role of gut microbiota in rheumatoid arthritis. J Clin Med [Internet]. 2017; 6. Available from: https:// www.ncbi.nlm.nih.gov/pmc/articles/PMC5483870/ (Accessed: 10.05.2019).

39. Tubbs AL, Liu B, Rogers TD, Sartor RB, Miao EA. Dietary salt exacerbates experimental colitis. J Immunol Baltim Md 1950 2017; 199: 1051-9.

40. Wyatt CM, Crowley SD. Intersection of salt- and immune-mediated mechanisms of hypertension in the gut microbiome. Kidney Int 2018; 93: 532-4.

41. Aguiar SLF, Miranda MCG, Guimarães MAF, et al. Highsalt diet induces IL-17-dependent gut inflammation and exacerbates colitis in mice. Front Immunol 2017; 8 : 1969.

42. Toussirot E, Béreau M, Vauchy C, Saas P. Could sodium chloride be an environmental trigger for immune-mediated diseases? An overview of the experimental and clinical evidence. Front Physiol 2018; 9: 440.

43. Monteleone I, Marafini I, Dinallo V, et al. Sodium chloride-enriched diet enhanced inflammatory cytokine production and exacerbated experimental colitis in mice. J Crohns Colitis 2017; 11: 237-45.

44. World Health Organization. Global health risks: mortality and burden of disease attributable to selected major risks. World Health Organization, Geneva 2009; 62.

45. Chobanian AV, Bakris GL, Black HR, et al. Joint National Committee on Prevention, Detection, Evaluation, and Treatment of High Blood Pressure. National Heart, Lung, and Blood Institute; National High Blood Pressure Education Program CoORdinating CommitTeE: Seventh 
report of the Joint National Committee on Prevention, Detection, Evaluation, and Treatment of High Blood Pressure. Hypertension 2003; 42: 1206-52.

46. Niklas A, Flotyńska A, Puch-Walczak A, et al. Prevalence, awareness, treatment and control of hypertension in the adult Polish population - Multi-center National Population Health Examination Surveys - WOBASZ studies. Arch Med Sci 2018; 14: 951-61.

47. Freeman AJ, Vinh A, Widdop RE. Novel approaches for treating hypertension. F1000Research [Internet]. 2017; 6. Available from: https://www.ncbi.nlm.nih.gov/pmc/ articles/PMC5288674/ (Accessed: 11.05.2019).

48. Garfinkle MA. Salt and essential hypertension: pathophysiology and implications for treatment. J Am Soc Hypertens 2017; 11: 385-91.

49. Nguyen H, Chiasson VL, Chatterjee P, Kopriva SE, Young KJ, Mitchell BM. Interleukin-17 causes Rho-kinase-mediated endothelial dysfunction and hypertension. Cardiovasc Res 2013; 97: 696-704.

50. Madhur MS, Lob HE, McCann LA, et al. Interleukin 17 promotes angiotensin II-induced hypertension and vascular dysfunction. Hypertension 2010; 55: 500-7.

51. Kamat NV, Thabet SR, Xiao L, et al. Renal transporter activation during angiotensin-II hypertension is blunted in interferon- $\gamma-/$ - and interleukin-17A-/- mice. Hypertension 2015; 65: 569-76.

52. Rodríguez-Iturbe B, Pons H, Quiroz Y, et al. Mycophenolate mofetil prevents salt-sensitive hypertension resulting from angiotensin II exposure. Kidney Int 2001; 59: 2222-32.

53. Machnik A, Dahlmann A, Kopp C, et al. Mononuclear phagocyte system depletion blocks interstitial tonicity-responsive enhancer binding protein/vascular endothelial growth factor $\mathrm{C}$ expression and induces salt-sensitive hypertension in rats. Hypertension 2010; 55: 755-61.

54. Mangrum AJ, Gomez RA, Norwood VF. Effects of AT(1A) receptor deletion on blood pressure and sodium excretion during altered dietary salt intake. Am J Physiol Renal Physiol 2002; 283: F447-53.

55. Crowley SD, Gurley SB, Oliverio MI, et al. Distinct roles for the kidney and systemic tissues in blood pressure regulation by the renin-angiotensin system. J Clin Invest 2005; 115: 1092-9.

56. Farquhar WB, Edwards DG, Jurkovitz CT, Weintraub WS. Dietary sodium and health: more than just blood pressure. J Am Coll Cardiol 2015; 65: 1042-50.

57. Sparks RL, Pool TB, Smith NK, Cameron IL. Effects of amiloride on tumor growth and intracellular element content of tumor cells in vivo. Cancer Res 1983; 43: 73-7.

58. Liu C, Zhu LL, Xu SG, Ji HL, Li XM. ENaC/DEG in tumor development and progression. J Cancer 2016; 7: 1888-91.

59. Litan A, Langhans SA. Cancer as a channelopathy: ion channels and pumps in tumor development and progression. Front Cell Neurosci 2015; 9: 86.

60. Rao VR, Perez-Neut M, Kaja S, Gentile S. Voltage-gated ion channels in cancer cell proliferation. Cancers 2015; 7: 849-75.

61. Treede I, Braun A, Jeliaskova P, et al. TNF- $\alpha$-induced up-regulation of pro-inflammatory cytokines is reduced by phosphatidylcholine in intestinal epithelial cells. BMC Gastroenterol 2009; 9: 53.

62. Murdaca G, Spanò F, Cagnati P, Puppo F. Free radicals and endothelial dysfunction: potential positive effects of TNF- $\alpha$ inhibitors. Redox Rep Commun Free Radic Res 2013; 18: 95-9.

63. White M. Mediators of inflammation and the inflammatory process. J Allergy Clin Immunol 1999; 103: S378-81.
64. Cassini-Vieira P, Araújo FA, da Costa Dias FL, et al. iNOS activity modulates inflammation, angiogenesis, and tissue fibrosis in polyether-polyurethane synthetic implants. Mediators Inflamm 2015; 2015: 138461.

65. Amara S, Tiriveedhi V. Inflammatory role of high salt level in tumor microenvironment. Int J Oncol 2017; 50: 1477-81.

66. Multhoff G, Molls M, Radons J. Chronic inflammation in cancer development. Front Immunol 2011; 2: 98.

67. Maldonado EN, Lemasters JJ. ATP/ADP ratio, the missed connection between mitochondria and the Warburg effect. Mitochondrion 2014; 19 Pt A: 78-84.

68. Amara S, Zheng M, Tiriveedhi V. Oleanolic acid inhibits high salt-induced exaggeration of Warburg-like metabolism in breast cancer cells. Cell Biochem Biophys 2016; 74: 427-34.

69. Peker KD, Ozkanli SS, Akyuz C, et al. Preoperative immunonutrition regulates tumor infiltrative lymphocytes and increases tumor angiogenesis in gastric cancer patients. Arch Med Sci 2017; 13: 1365-72.

70. Pidgeon GP, Barr MP, Harmey JH, Foley DA, BouchierHayes DJ. Vascular endothelial growth factor (VEGF) upregulates $\mathrm{BCL}-2$ and inhibits apoptosis in human and murine mammary adenocarcinoma cells. Br J Cancer 2001; 85: 273-8.

71. Amara S, Alotaibi D, Tiriveedhi V. NFAT5/STAT3 interaction mediates synergism of high salt with IL-17 towards induction of VEGF-A expression in breast cancer cells. Oncol Lett 2016; 12: 933-43.

72. Pan B, Shen J, Cao J, et al. Interleukin-17 promotes angiogenesis by stimulating VEGF production of cancer cells via the STAT3/GIV signaling pathway in non-small-cell lung cancer. Sci Rep 2015; 5: 16053.

73. Knochelmann HM, Dwyer CJ, Bailey SR, et al. When worlds collide: Th17 and Treg cells in cancer and autoimmunity. Cell Mol Immunol 2018; 15: 458.

74. Shikata K, Kiyohara Y, Kubo M, et al. A prospective study of dietary salt intake and gastric cancer incidence in a defined Japanese population: the Hisayama study. Int J Cancer 2006; 119: 196-201.

75. WHO. Sodium intake for adults and children [Internet]. Available from: https://www.who.int/nutrition/ publications/guidelines/sodium_intake/en/ (Accessed: 9.05.2019). 